\section{NP30 School Dining Redesign: Impacts on Students and Teachers in Middle and High Schools in California}

Lorrene Ritchie, PhD, MS, RD, lritchie@ucanr.edu, University of California, Division of Agriculture and Natural Resources, Nutrition Policy Institute, 2115 Milvia Street, Suite 301, Berkeley, CA 94704; Annie Reed, MPP, University of California, Berkeley, School of Public Health; Kellie Ottoboni, BS; Hannah Thompson, PhD, MPH; Marissa Neelon, MS, RD, UC Cooperative Extension, Contra Costa County; Kris Madsen, MPH, MD, University of California, Berkeley, School of Public Health

Objective: The purpose of the study was to evaluate a school dining redesign in a large school district in California. Outcomes included school lunch participation, student intake of fruits and vegetables, and teacher promotion of school meals.

Description: Using a quasi-experimental controlled trial design, we present results from the first year of a 2-year implementation of a 3-pronged intervention: dining room redesign, distributed points of sale of school lunch, and staff promotion of school meals. Linear regression models were used to compare changes from 2016 to 2017 in survey responses from 7 th-10th grade students and teachers from 12 middle and high schools compared to 12 control schools. Models of student survey data were adjusted for race/ ethnicity, gender and grade. Changes from 2016-2017 in school lunch participation were adjusted for school enrollment, free and reduced price meal enrollment, and student race/ethnicity.

Evaluation: Surveys were collected from over 8,000 students and over 500 teachers. Half of students were Asian, 17\% Latino, 10\% White, 4\% African-American. Significantly more students in intervention compared to control schools reported eating school breakfast and getting lunch from a mobile school cart. More intervention students indicated that they liked the fruit served with lunch, staff encouraged them to eat school lunch, and the school lunch was healthier than foods from elsewhere. Compared to teachers at control schools, teachers at intervention schools reported more often eating the school lunch, and eating from the healthy student vending machines as opposed to the traditional staff vending machines. No increases in the weekly frequency of students eating fruits and vegetables or in teachers promoting school meals to students were detected. Trends in school lunch participation appear promising.

Conclusions and Implications: Preliminary outcome data suggest that a school dining redesign may be effective in improving student perceptions of school meals and participation in school lunch. Future analyses will include 2-year results and assessment of school meal waste.

Funding: 2015-68001-23236.

\section{NP31 Starting Early/Empezando Temprano: Randomized Control Trial to Test the Effectiveness of a Child Obesity Prevention Program}

Mary Jo Messito, MD, mary.messito@nyumc.org, NYU School of Medicine Bellevue Hospital, 462 First Avenue, New York, NY 10016; Alan Mendelsohn;

Roberta Scheinmann, MS; Rachel Gross, MS, MD

Objective: To improve maternal infant feeding and activity practices and reduce child obesity with the Starting Early Program (StEP): a primary care-based obesity prevention intervention beginning in pregnancy for lowincome Hispanic mother-infant dyads.

Description: Conduct a randomized control trial of the StEP intervention. Women enrolled in the third trimester of pregnancy at an urban public hospital clinic, randomized to StEP intervention vs. standard primary care. Intervention included: individual nutrition counseling in prenatal/peri-partum periods, nutrition and parenting support groups at every well child visit with 2 individual and 13 group intervention sessions scheduled prior to child age 3 years. Feeding styles, diet and lifestyle patterns, psychosocial stressors, and demographic information collected at baseline in pregnancy and infant age 3, 10, 19, months, 2 and 3 years, with measured length/height and weight. Multilevel modeling used to assess intervention impact on infant weight for length z-scores (WFLz). T tests and chi square used to assess impact on nutrition knowledge and practices. Logistic regression used to assess intervention dose on risk of obesity (BMI $\geq 95$ th\% for age and sex) at age 3 .

Evaluation: Baseline prenatal assessments $(n=566)$ found high rates of maternal stress, obesity and unhealthy dietary practices. Five hundred and thirty three pregnant women randomized to StEP vs. standard care. No group differences in baseline mother/infant characteristics. Mean sessions attended by 3 years: 8.5 (range 1-15). At infant age 3 months: higher nutrition knowledge, higher rates of exclusive breastfeeding, tummy time and less early introduction of complimentary foods for intervention group. At 10 months, more exclusive breastfeeding, more responsive feeding styles and less introduction of juice. At age 2, more family meals and less restaurant food. Complete weight data available for infants through age 18 months $(n=433)$. Intervention group infants had lower mean WFLz between ages 12-18 months (-.21 (.10), $\mathrm{p}=.046)$. At 36 months, infants attending $>10$ sessions were less likely to have obesity, $\mathrm{p}=.039$.

Conclusions and Implications: Interventions like StEP have the potential to improve maternal infant feeding and activity practices and reduce childhood obesity.

Funding: 2011-68001-30207. 